\title{
THE PATCHWORRK ARRAM
}

\author{
After years of delays and cost overruns, an \\ international collaboration is finally inaugurating \\ the world's highest-altitude radio telescope.
}

BY ERIC HAND

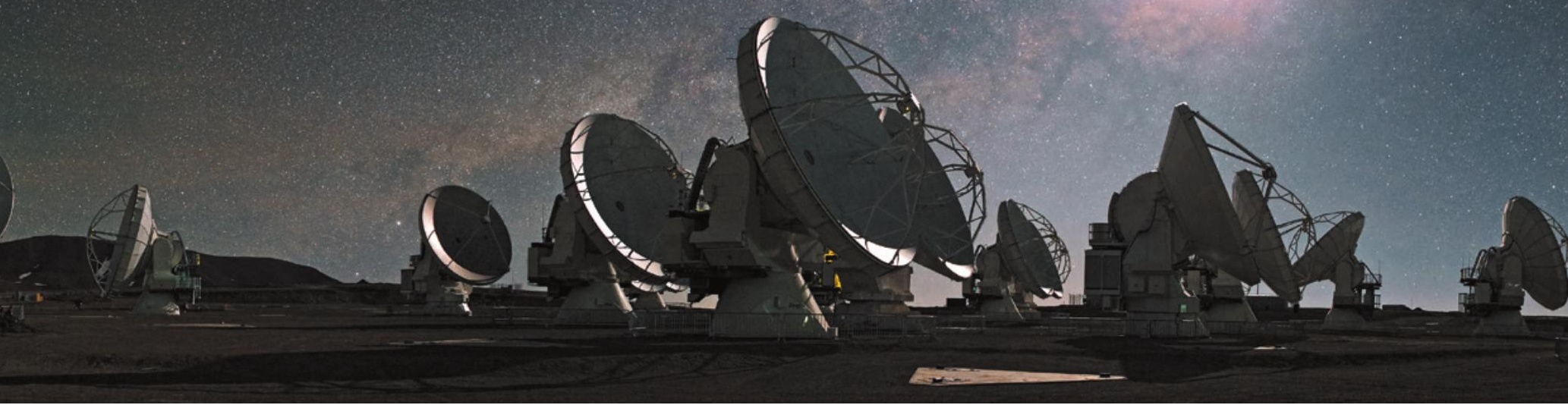

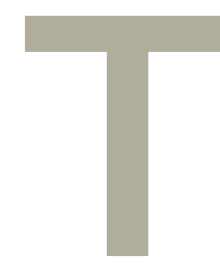

he car toils upwards along the sinuous road, its engine tuned for the thin air. The clumps of cactus and grass along the road soon give way to bone-dry lifelessness. By the time the car reaches 4,000 metres above sea level, Pierre Cox has a bit of a headache. By the time it reaches the 5,000-metre-high Chajnantor plateau - one of the highest, driest places on Earth, and one of the best for astronomy - the altitude is affecting his bladder. Cox, the incoming director of the Atacama Large Millimeter/submillimeter Array (ALMA) in Chile, is about to glimpse the giant telescope dishes he will soon be responsible for. But first he must find a toilet.

Cox slides out of the car and staggers into ALMA's glass and steel operations centre. The current director, Thijs de Graauw, a trim 71-year-old Dutchman, follows Cox inside and sits down. For him, journeys like this occur weekly — if not daily — but he knows that they are no joke. First-timers get a mandatory medical screening before being allowed up to the plateau, and regular shift workers pad around the building with tubes in their noses and oxygen tanks on their backs. "Everyone okay?" De Graauw asks the group of astronomers who have accompanied Cox to ALMA on this December day. "No victims yet?"

Cox re-emerges from the toilet, puts on wraparound sunglasses and, slightly dizzy, heads outside with the group. Scattered across the surrounding plain of brown volcanic soil are dozens of huge white radio antennas, looking as out of place as the stone statues on Easter Island. High on this cold and lonely plateau, they are gathering photons from the cold and lonely parts of the Universe - the dimly glowing clouds of dust and gas where stars are born. Their signals are then combined into images that have a resolution better than that of the Hubble Space Telescope.

The stillness of the tableau breaks as the dishes begin to tilt and swivel in unison. "My goodness," says Cox, hushed by the sight of so much metal moving so quickly and quietly.

But the choreography is not quite uniform. Clustered tightly in the middle of the array are 12 dishes, each 7 metres across, and four 12-metre dishes, from Japan. Spaced farther out are 25 dishes, each 12 metres across and fitted together like pie slices, from the United States. And scattered among those are the first of 25 dishes from Europe, each 12 metres across top-of-the-line carbon-fibre devices pivoting on silky-smooth gearing.

The last of those European antennas will not be installed until the end of 2013, when ALMA will finally reach its full complement of 66 dishes. Rather than wait until then, however, the project held a formal inauguration ceremony on 13 March to celebrate the collaboration that made it all possible. A total of 19 countries have contributed to ALMA, through

three primary partners: the European Southern Observatory (ESO); the National Astronomical Observatory of Japan; and the US National Radio Astronomy Observatory (NRAO) in Charlottesville, Virginia, funded by the US National Science Foundation (NSF).

Less celebrated have been the difficulties of keeping this unwieldy confederation on track - with power shared among three independent organizations that have different cultures and norms. Nor is anyone likely to cheer about how the lack of unity caused the US\$1.4-billion project to come in several years late, well over cost and downsized from its original ambitions. Its successive directors have had to be diplomats and negotiators as much as scientists.

But ALMA is not unique in that respect. International mega-projects are becoming increasingly common in astronomy. Witness the Square Kilometre Array, a proposal to build 3,000 radio dishes with a total collecting area approaching 1 square kilometre in Australia and South Africa (see Nature 484, 154; 2012). As costs for such ambitious projects cross the billion-dollar threshold, nations are finding that they cannot go it 


\section{'I think it's the largest science project ever where nobody was in charge, but
we have made it work." nobody was in charge, but
we have made it work."}

alone - a situation for which ALMA might serve as a valuable object lesson. "I think it's the largest science project ever where nobody was in charge," says Ethan Schreier, president of Associated Universities Incorporated (AUI), a radio-astronomy research-management company based in Washington DC, which operates the NRAO. "But we have made it work."

\section{FAMILY TIES}

Each of the three primary partners came to Chile in its own way, with pilot projects dating from the 1980s. Of particular interest for Europe was the infrared glow from the dust that shrouds many of the Universe's first galaxies. This glow can be used to estimate the size, brightness and number of stars hidden within - key questions for astronomers trying to piece together the history of galaxy formation. Shifted to longer wavelengths by the expansion of the Universe, this glow reaches telescopes on Earth as millimetre-wave radiation - and can be detected day or night, as long as there isn't much atmospheric water vapour in the way.

To get at the earliest (and thus most distant and faint) of these dusty galaxies, European astronomers needed a large collecting area. They proposed an array of 16-metre dishes on the salt flats of the Atacama Desert, more than 1 kilometre lower than the Chajnantor plateau. US astronomers were more interested in star formation within our Galaxy, and wanted the better image quality that would come with an array of 8-metre dishes placed more closely together. They also wanted to push into the shorter wavelengths of the unexplored submillimetre band, where they could study chemical-emission lines from molecules in interstellar gas clouds. They pushed hard for Chajnantor, which was high and dry enough for submillimetre observations, and flat enough for the dishes to be moved into various configurations.

Pooling resources was an obvious move for the two projects, and in 1997, ESO director Riccardo Giacconi and NRAO director Paul Vanden Bout signed a joint resolution to pursue a compromise - a facility of 64 dishes, each 12 metres across. "Riccardo and I signed this document with no authority whatsoever," says Vanden Bout. The official backing from the ESO and the NSF wouldn't come for another six years.

Japan joined the partnership in 2004 and committed to building 16 dishes in the centre of the array. The more widely spaced US and European dishes would provide high-resolution detail in a narrow field of view. But the compact array would give a more complete view of large objects such as the Galactic Centre or the sprawling, dusty clouds where the Milky Way forms its new stars.

The patchworked nature of ALMA's creation is reflected in its organizational structure.
Partner agencies have been loath to relinquish control over budgets (or anything else), so the coordinating body that manages array operations - the Joint ALMA Observatory (JAO) - has no formal authority. For example, when Chile created a science preserve on the Chajnantor plateau (in return for $10 \%$ of ALMA's observing time), officials signed the lease with AUI and the ESO, not the JAO.

\section{CULTURAL SENSITIVITY}

ALMA directors quickly learn that management works best through persuasion, not proclamation. "You have to seduce," de Graauw says. Cultural sensitivity is also required. On conference calls, de Graauw says, his Japanese colleagues would say nothing until he solicited them directly for comments. Alison Peck, deputy project scientist for ALMA, learned a similar lesson about setting deadlines. "In Japan, it's really not okay to miss a deadline," she says. "In the United States, you can usually make reasonable excuses and ask for an extension. In Europe they worry about it even less."

ALMA's motley nature is apparent even in the 12-metre telescope dishes, the array's biggest single cost. From the beginning, the technical requirements were "truly daunting”, says Tony Beasley, a former ALMA project manager and current head of the NRAO. Each dish needed a motor that could accurately point at celestial targets to within 
0.6 arcseconds (about the same apparent size as a bacterium at arm's length); a reflecting surface with an accuracy of 25 micrometres (about one-quarter of the width of a human hair); and structural materials that could maintain that precision in the face of Chajnantor's wicked winds and subzero temperatures.

The cheapest way to meet those requirements would have been for the ESO and the NRAO to share a single design and a single contractor. But the NRAO went with a small US firm Vertex, which was later bought by General Dynamics of Falls Church, Virginia - and the ESO held out for a European consortium led by Thales Alenia Space, based in Paris. The delays associated with going to separate contracts came just as prices for commodities such as steel were rocketing because of demand in China, leading to a dramatic escalation in ALMA's cost. As a result, in 2005, the project was 'descoped' - the NRAO and the ESO would each contribute only 25 antennas rather than 32 , resulting in a loss in array sensitivity (see Nature 439, 526-528; 2006). Even with the descope, US and European contributions to ALMA would grow from $\$ 650$ million to $\$ 1$ billion.

Japan, meanwhile, had contracted its dishes to Mitsubishi Electric, based in Tokyo. The three companies maintained separate assembly sites at the ALMA operations support facility (OSF), a cluster of buildings where most staff members live and work. (The OSF was built at 2,900 metres, in part because it costs less to hire Chilean workers for altitudes lower than 3,000 metres.)

It is too early to tell whether one design will outperform the others. The ESO's carbonfibre dishes change pointing position with fewer errors, but it is uncertain how well the advanced internal gearing will hold up to weather over time. So far, all the antennas are performing to specifications. But having three different designs will saddle ALMA with extra operations costs far into the future, says Neal Evans, an astronomer at the University of Texas at Austin and chair of the ALMA board. "You'll need different spare parts, and you'll need people that know how to maintain each of the designs," he says.

\section{AMBITIOUS TARGETS}

Despite all the headaches, antennas are steadily accumulating on the plateau. In 2007, the JAO team raised glasses of water to celebrate the first linking of two dishes using the correlator - a computer that connects dish signals to create a composite view of the sky. (Why no champagne? The altitude impairs judgement, even at 2,900 metres, so ALMA has a strict no-alcohol policy; workers are subject to random breathalyser tests on the buses connecting local towns to the OSF.)

In September 2011, with 16 dishes in place, ALMA began its inaugural observing period with the 100 or so projects that had risen to the top of its 'cycle 0 ' proposal competition. Most of

\section{ALMA, SMALL AND LARGE}
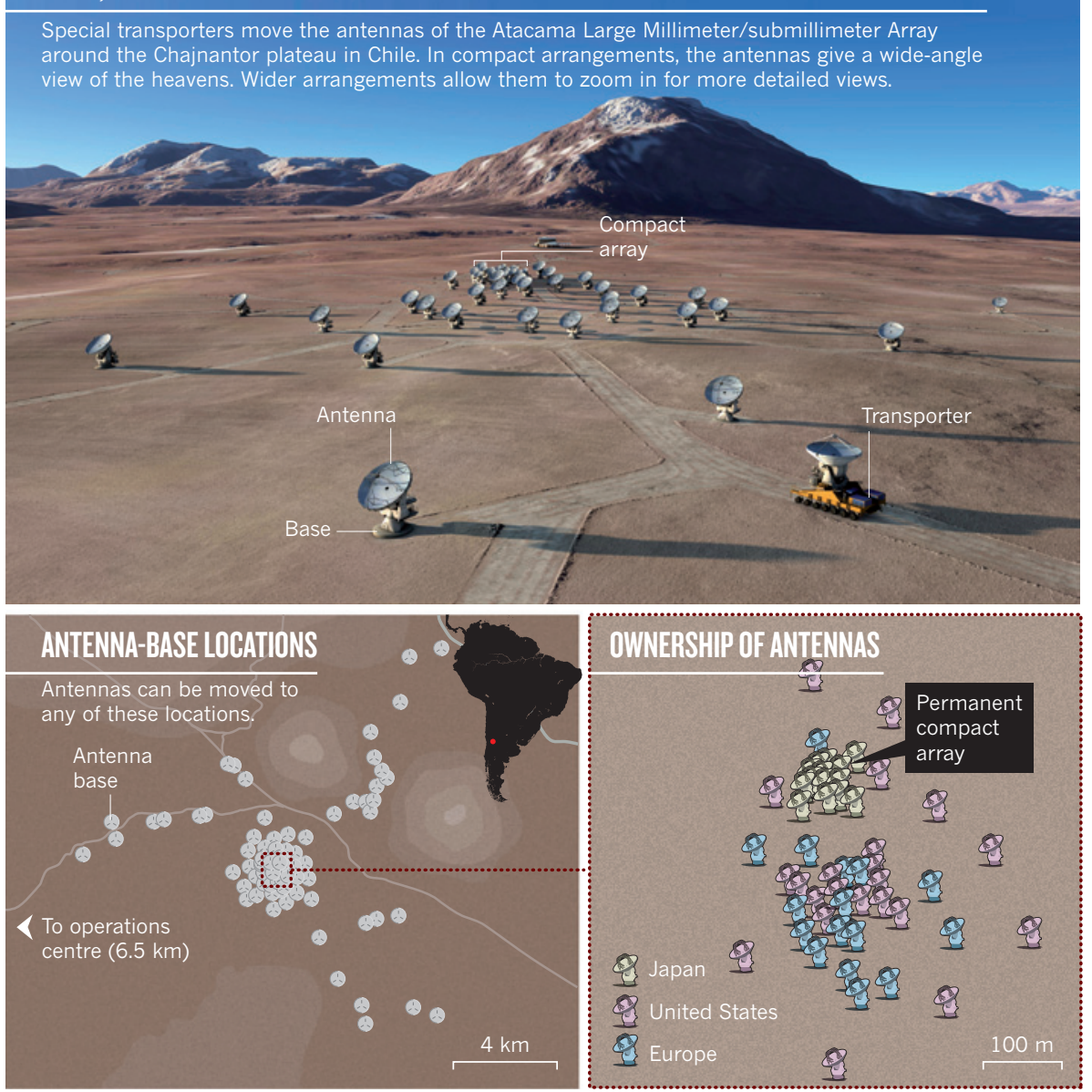

the observation targets were relatively nearby objects in our Galaxy. Results ranged from the detection of sugar-related molecules in a nearby star system to an exceptionally sharp image of the gas clumps that will collapse into giant stars (see Nature 492, 319-320; 2012).

But the targets will soon become more ambitious. The mathematics of radio arrays implies an inverse relationship between antenna spacing and image resolution: the longer an array's 'baselines' (the distances between pairs of antennas), the smaller its field of view and the higher its resolution. The number of baselines, which determines how 'filled in' an ALMA image is, has grown simply through the addition of antennas. But the observatory can also change baselines by moving the antennas around the plateau - with the help of two German-built transporters nicknamed Otto and Lore (see 'ALMA, small and large').

In January, ALMA began its cycle 1 observations with 32 of the 12-metre dishes working at baselines of up to 1 kilometre, combined for the first time with some of the smaller Japanese dishes in the centre of the array. By the time cycle 2 begins, in early 2014, ALMA astronomers hope to have 40 dishes working at baselines of up to 2 kilometres. The resulting high resolution will help astronomers to understand star formation in distant galaxies seen very early in their lives, when the Universe was young and its chemical composition was different. "ALMA could very well open up a whole new field of star formation," says Linda Tacconi, an astronomer at the Max Planck Institute for Extraterrestrial Physics in Garching, Germany.

ALMA will also be able to pinpoint how far away, and therefore how old, an object is. Usually, that measurement is a two-step process. Researchers first need time at a radio-astronomy facility to locate the object - a distant galaxy, say - then must spend many hours on an optical telescope to split the faint light up into its spectral components and identify emission or absorption lines caused by the presence of various elements and molecules. Measuring how far those wavelengths have been stretched by cosmic expansion allows observers to estimate how far away the object is.

ALMA can do all of the above within minutes. Already, ALMA observations have shown that strangely bright early galaxies were in fact multiple smaller galaxies that had been lumped together by an earlier optical survey (A. Karim et al. Preprint at http://arxiv. org/abs/1210.0249; 2013). The discovery was a relief to theorists, who had been unable to work out how such bright, huge galaxies could have formed so early in the Universe.
DNATURE.COM To read more about science at ALMA, visit: go.nature.com/cv5btp 
Beasley says that it would be better to create a strong central authority at the outset, and persuade funding agencies to grant it budgetary powers. There are precedents, particularly the treaty-governed European research institutes such as CERN, a particlephysics facility in Geneva, Switzerland, and the ESO itself, whose member states pay dues each year. And in 2011, the Square Kilometre Array created the SKA Organisation - a non-profit company based at the Jodrell Bank Observatory near Manchester, UK - which might give it the authority missing from the JAO.

But it is hard to imagine a funding agency such as the NSF - which answers to the US Congress - ceding control. So in the near term, big astronomy is likely to be governed by loose confederations, and the success of future mega-projects will depend on the savvy and sweat of the people within. Anyone who has served as ALMA director would know something about this. Each charmed rather than shouted his way to success. De Graauw had his courtliness; Massimo Tarenghi, director from 2003 to 2008, a certain puckishness. Cox's weapon might be positivity: the new director seems always to be grinning. Tarenghi hopes that those smiles will stay after Cox takes the helm in April. "The person that suffers most is the poor director," he says wryly.

\section{COMING DOWN THE MOUNTAIN}

By the end of the one-hour tour of ALMA, Pierre Cox is in fact suffering - from oxygen deprivation. Yet he still seems to be on cloud nine. "I'm infinitely grateful. I'm honoured. I'm thrilled," he says. "This is one of the coolest places I've ever been.” He gets in the car for the downhill journey and slips an oxygen saturation meter over his finger First it reads $70 \%$, then $76 \%$. Not good. The driver, who has already put oxygen tubes in his own nose, calmly hands Cox a pressurized can of oxygen. Cox takes a squirt in his mouth and checks his numbers again. More than $90 \%$. Much better.

Maybe it's the rush of oxygen to the brain, but Cox becomes an enthusiastic chatterbox. The high-redshift Universe will be just the beginning, he says. He won't be completely satisfied following up on the objects others have already spotted. "There will always be surprises," he says. The car passes a vicuña (a relative of the llama) standing sentinel at the lip of a gully. ALMA's dishes have vanished behind the edge of the plateau. The OSF appears in the distance below, white rooftops shimmering as the desert heats up for the day. By 4,000 metres, the air is getting thicker. The oxygen has a soporific effect. De Graauw's head begins to nod. Cox yawns loudly. Inexorably, his eyelids close.

Behind him, on an isolated plain at the top of the world, the eyes of ALMA remain open, alert to the earliest glimmers of the Universe.

Eric Hand is US news editor for Nature.

The question is whether the JAO is strong enough to marshal ALMA's partners to do a deep-field survey. The Hubble Space Telescope decades - but only because Hubble directors have allocated large chunks of discretionary the fierce competition for observation ALMA directors have very little discretion been allocated. partners would have to donate the time x proposals for each available slot.

The problem highlights a complaint comcan call the shots. Europe and the United States no one has a majority. "There's no tiebreaker"

Yet Beasley doubts that the process w have been any smoother if Europe or the United States had taken the lead. For smaller projects, he says, with stakes on the order of millions of dollars, minority funding partners might accept some decisions that run counter to their interests. But with a project the size of ALMA, in which even a minority stake is hundreds of millions of dollars, funding agencies will fight to protect their interests. "No one is going to lose any significant decisions at that point," he says. 